\title{
Las invasiones vegetales en sistemas agrícolas. Retrospectiva de los últimos 40 años en Cataluña
}

\author{
J. Recasens ${ }^{1, *}$, J.A. Conesa ${ }^{1,2}$ y A. Juárez-Escario ${ }^{1}$
}

1 Grupo de Malherbología y Ecología Vegetal. Agrotecnio. Departamento de Hortofruticultura, Botánica y Jardinería. Escola Tècnica Superior d'Enginyeria Agrària. Universitat de Lleida

2 Arborètum-Jardí Botànic de Lleida. Calle Enric Farreny 49. 25199 Lleida. Av. Alcalde Rovira Roure 191. 25198, Lleida

\begin{abstract}
Resumen
Los sistemas agrícolas son una de las vías preferentes de introducción de especies vegetales exóticas y la perturbación asociada a estos hábitats ha favorecido muchos casos de invasión. Sin embargo, el estudio de este proceso ha recibido menor interés que en sistemas naturales o seminaturales. Este hecho parece deberse a una desigual visión acerca del impacto causado en el equilibrio ecológico del sistema. En el presente artículo se realiza una revisión de las causas intrínsecas que favorecen los procesos de invasión y los rasgos que definen la invasibilidad de los sistemas agrícolas. Como ejemplo se utilizan los diferentes casos registrados en los últimos 40 años en Cataluña y sobre los que se dispone de amplia información. Se exponen las diferentes tipologías de cultivo preferentemente sensibles a acoger malas hierbas exóticas y las características ambientales y estrategias de manejo a las cuales estas especies han mostrado mejor adaptación. Los principales escenarios agrícolas que se definen son: campos de arroz, campos de maíz y otros cultivos anuales de regadío, campos de alfalfa y cultivos leñosos de regadío. Se recogen también las distintas disposiciones legislativas existentes al respecto a nivel de Cataluña, exponiendo de manera crítica, las diferencias de criterio utilizadas entre las listas de alerta publicadas a nivel europeo, nacional y autonómico, respecto a las especies que constituyen problema o amenaza en los campos de cultivo.
\end{abstract}

Palabras clave: Malas hierbas exóticas, cultivos, ecología, legislación, impacto.

Plant invasions in agricultural systems. Retrospective of the last $\mathbf{4 0}$ years in Catalonia

\begin{abstract}
Agricultural systems are one of the preferred ways of introduction of exotic weed species and the disturbance associated with these habitats has favored many cases of invasion. However, the study of this process has received less interest than in natural or semi-natural systems. This fact seems to be due to an unequal view about the impact caused on the ecological equilibrium of the system. In this article, a review of the intrinsic causes that favor the weed invasion processes and the features that define the invasiveness of agricultural systems is carried out. As an example, the different cases registered in the last 40 years in Catalonia, and for which a lot of information is available, are used. The different crop typologies preferably sensitive to host exotic weeds and the environmental characteristics and management strate-
\end{abstract}

\footnotetext{
* Autor para correspondencia: jrecasens@hbj.udl.cat
}

Cita del artículo: Recasens J, Conesa JA y Juárez-Escario A (en prensa). Las invasiones vegetales en sistemas agrícolas. Retrospectiva de los últimos 40 años en Cataluña. ITEA-Información Técnica Económica Agraria. Vol. xx: 1-22. https://doi.org/10.12706/itea.2019.020 
gies to which these species have shown better adaptation are exposed. The main agricultural scenarios that are defined are: rice fields, corn fields and other annual irrigated crops, alfalfa fields and irrigated orchards. It also includes the different existing legislative rules in this regard at the level of Catalonia, exposing critically, the differences in criteria used between the alert lists published at European, national and regional level, with respect to the species that constitute a problem or threat in the farmlands.

Keywords: Exotic weeds, crops, ecology, legislation, impact.

\section{Introducción}

En las últimas décadas, el fenómeno de las invasiones biológicas ha tomado gran notoriedad tanto desde el punto de vista ecológico como social. No en vano, se considera como uno de los principales elementos transformadores de las comunidades biológicas, alterando hábitats y amenazando la supervivencia de especies nativas (Pyšek et al., 2009). Aunque el impacto y costes del control de especies vegetales exóticas en sistemas agrícolas resulta mayor que en cualquier otro escenario, ha recibido un menor interés que el causado en sistemas naturales o seminaturales (Daehler, 1998). De hecho, existe una visión dual en la definición de especie invasora. Para algunos autores (Richardson et al., 2000; Pyšek et al., 2004) la definición excluye cualquier alusión al "impacto" causado, y se centra exclusivamente en criterios ecológicos y biogeográficos. Por el contrario, la definición dada por diversas organizaciones internacionales (IUCN, Convention on Biological Diversity, World Trade Conservation) asumen de forma explícita el impacto causado por esas especies en la economía, el medio ambiente y en la salud (IUCN, 2000). Además, según Clements et al. (2004), los procesos de invasión en paisajes agrícolas por parte de especies que expanden su área geográfica, es un proceso continuo donde puede aplicarse, al igual que en sistemas naturales, la teoría ecológica de las especies invasoras.

No debemos olvidar que los sistemas agrícolas son una de las vías preferentes de introducción de especies exóticas. Según Pyšek et al. (2009) un $63 \%$ de las especies vasculares naturalizadas en Europa lo han sido a través de introducciones voluntarias, siendo un 58 $\%$ a partir de su llegada como ornamentales o cultivos de interés hortícola y el $5 \%$ restante como contaminantes de semillas. Por ello, los sistemas agrícolas constituyen uno de los escenarios más favorables, sino el más importante, para la introducción y persistencia de especies vegetales exóticas.

A nivel europeo, la zona mediterránea es la que ha mostrado, en las últimas décadas, un mayor y drástico cambio a nivel de paisaje agrícola. La conversión de zonas de secano en regadío ha permitido incrementar los niveles de humedad del suelo y la disponibilidad de nutrientes $y$, con ello, contribuir a un importante cambio en la composición de las comunidades arvenses (Juárez-Escario et al., 2013). En Cataluña, los nuevos planes de regadío implementados en las dos últimas décadas, no sólo han permitido incrementar la superficie de cultivo sino también su intensificación. En un estudio reciente, Juárez-Escario et al. (2018) describen cómo el manejo de los campos de maíz y frutales de regadío en los últimos 20 años ha influido en la composición y tipos funcionales de las comunidades arvenses, y cómo dichos cambios han beneficiado a ciertas especies exóticas en detrimento de la flora arvense nativa. Dado que los sistemas agrícolas constituyen escenarios altamente sensibles a la invasibilidad realizamos en este artículo un análisis, tanto desde el punto de vista ecológico como legislativo, de los casos registrados en los últimos 40 años en distintos cultivos de Cataluña. 


\section{Implicaciones agronómicas}

Los agroecosistemas constituyen hábitats favorables a la presencia de flora exótica (Vilà et al., 2004; Pyšek et al., 2009) pudiendo ésta ejercer un elevado impacto tanto a nivel económico como agronómico. Debido al aumento del comercio internacional de semillas y productos agrícolas, este proceso se ha visto, en las últimas décadas, incrementado de forma significativa y constituye un claro exponente del cambio climático, al favorecer la naturalización de muchas especies de origen tropical (Sanz-Elorza et al., 2004). En cuanto a impacto económico, y a modo de ejemplo, sólo en Estados Unidos las pérdidas ocasionadas por la presencia de flora invasora en sistemas agrícolas se han estimado en $\mathbf{2 7 . 0 0 0}$ millones de dólares anuales (Pimentel et al., 2005), teniendo en cuenta tanto la disminución del rendimiento de las cosechas como los costes de control implementados. A este impacto económico causado por las especies invasoras, podemos sumar los impactos ambientales que representan las medidas de control como la aplicación de herbicidas (Vilà et al., 2004) o el impacto que dichas especies pueden tener sobre las comunidades naturales incluidas en la matriz de los agroecosistemas (Juárez-Escario et al., 2016). A su vez, la presencia de malas hierbas invasoras puede provocar una reducción en los recursos genéticos de la flora nativa (Pál, 2004), la formación de nuevos ecotipos mediante la hibridación con especies nativas, o producir una disrupción de la estructura de las comunidades arvenses autóctonas (Protopopova et al., 2006). Como resultado del dominio de las especies exóticas, se puede favorecer la homogeneización de las comunidades arvenses con el impacto negativo que puede suponer en los servicios ecosistémicos que éstas proveen (Juárez-Escario et al., 2018).

\section{Ecología de las invasiones en el marco de los agroecosistemas}

En todo proceso de invasión biológica existen una serie de etapas clave (Richardson et al., 2000). Para que una especie alóctona devenga invasora, primero debe ser introducida en un nuevo hábitat y mediada por la actividad antrópica. En los hábitats agrícolas, las principales vías de introducción involuntaria de especies exóticas son la contaminación de lotes de semillas no certificados, la dispersión mediada por el manejo del propio cultivo (p.e. a través de la maquinaria agrícola) o la dispersión involuntaria de especies con interés ornamental o forrajero (Sanz-Elorza et al., 2009). Si una especie está presente de forma esporádica o casual, pero sin haberse establecido aún, se le denomina adventicia. Si, por el contrario, ha establecido poblaciones, ello implica que es capaz de reproducirse y formar colonias autoperpetuantes, en un proceso definido como colonización. Finalmente, si la especie colonizadora es capaz de dispersarse y generar por sí misma nuevas poblaciones y superar una serie de filtros ambientales, se le denomina, en ecología, como especie naturalizada, y desde el punto de vista de los impactos que provocan, se le califica como especie invasora (Richardson et al., 2000).

Una vez que una especie exótica se ha introducido en un hábitat concreto, el éxito en establecer poblaciones estables a partir de las cuales poder dispersarse depende de los siguientes factores: a) las características intrínsecas de la especie para llevar a cabo este ciclo de forma exitosa (lo que se ha denominado capacidad invasora); b) la propia susceptibilidad de los hábitats a ser colonizados por dichas especies (invasibilidad del hábitat, que engloba tanto las características climáticas de la región hospedadora como las particularidades ambientales del hábitat hospedador), y c) factores tales como el tiempo de residencia de la especie o la presión de llegada de propágulos ejercida (Richardson y Pyšek, 2006). 
La capacidad invasora de las especies viene definida por la posesión de una serie de atributos que les confieren una ventaja adaptativa en el medio donde se instalan. Atributos tanto vegetativos como reproductivos relacionados con la adquisición y almacenamiento de recursos y con la capacidad de colonización. En el caso de sistemas agrícolas, particularmente los de regadío, se han descrito para especies vegetales y como atributos relacionados con una mayor capacidad invasora las formas biológicas graminoides, la fotosíntesis C4, la clonalidad y la hidrocoria (Juárez-Escario et al., 2013), si bien otros atributos, como la producción de un elevado número de semillas y su elevada viabilidad también están detrás de su éxito invasor (Recasens y Conesa, 2003). En los agroecosistemas, la selección de dichos atributos está ligada al manejo que se lleva a cabo en los cultivos. Prácticas como la realización de labores o siegas suponen importantes perturbaciones, que ejercen una fuerte presión de selección sobre las especies de malas hierbas. A su vez, la aplicación de herbicidas añade aún mayor presión de selección sobre la flora arvense, llegando a favorecer incluso la aparición de biotipos resistentes a herbicidas.

En hábitats fértiles, ricos en nutrientes y sometidos a perturbaciones periódicas, el éxito de las especies exóticas se ha relacionado con atributos funcionales diferentes de aquellos que dominan entre las comunidades locales y que les permiten una preadaptación al medio y un crecimiento y dispersión rápidos (Hejda y de Bello, 2013).

En cuanto a la invasibilidad de los hábitats, la susceptibilidad a ser colonizados por especies invasoras se puede definir en términos del nivel de perturbaciones que soportan y la disponibilidad de nutrientes (Pyšek et al., 2010). Un ecosistema sometido a perturbaciones periódicas dispone de nuevos nichos donde el nivel de competencia interespecífica es muy bajo y la disponibilidad de nutrientes es ele- vada (Shea y Chesson, 2002) lo que genera oportunidades para la entrada y naturalización de especies exóticas. A su vez, en estos escenarios, la posibilidad de éxito de las especies exóticas es mayor si son capaces de aprovechar el incremento de dichos recursos de manera más efectiva que las especies nativas (Davis et al., 2000).

Otro factor relevante en los procesos de invasión lo constituye la presión de propágulos, es decir la cantidad de efectivos que se introducen en un momento y hábitat concretos, así como el número de eventos de introducción. Una presión de propágulos sostenida en el tiempo influye notablemente en el potencial establecimiento de una planta exótica (Richardson y Pyšek, 2006). En dicho proceso también entran en juego los patrones espaciales, ya que los hábitats conectados o cercanos a la fuente de invasión son los más propensos a recibir una mayor presión de propágulos de especies exóticas. En ambientes agrícolas, dicha presión está intensificada por la actividad humana, a través de la maquinaria agrícola o el riego por inundación. Estas características del manejo agrícola suponen que la escala espacial y temporal donde la presión de propágulos es más efectiva sea mucho mayor que en hábitats naturales, lo que aumenta la probabilidad de una expansión rápida y eficaz de las especies introducidas.

\section{Procesos de invasión más singulares registrados en los sistemas agrícolas de Cataluña}

Haciendo una retrospectiva de los principales casos de invasiones vegetales habidas en los sistemas agrícolas en Cataluña, podemos constatar que éstas han tenido lugar en diferentes tipologías de cultivos de acuerdo a unas pautas concretas de manejo en cada uno de ellos. Por ello los casos de éxito en cuanto a presencia y expansión de ciertas especies debe relacionarse tanto con atributos biológicos de 
las especies como en su adaptabilidad a las pautas de manejo que rigen en esos cultivos. Las cuatro tipologías de escenarios agrícolas propuestos son: a) campos de arroz; b) campos de maíz y otros cultivos anuales de regadío; c) alfalfa y otros cultivos forrajeros y d) cultivos leñosos de regadío. Aparte de la perturbación implícita al propio manejo del cultivo, existe un denominador común en todos ellos que define su invasibilidad, como es la estacionalidad estival y la presencia de riego. Estos dos factores combinados con los atributos de las especies y su adaptación al manejo favorecen los casos de invasión.

A continuación, se exponen, para cada tipología de cultivo propuesto, los principales casos de invasiones vegetales registrados y las causas que han favorecido la entrada y expansión de esas malas hierbas exóticas invasoras.

\section{Campos de arroz}

La flora arvense de los campos de arroz se corresponde con unos rasgos biológicos y ecológicos característicos relacionados con las singulares características del hábitat y su manejo. La hidrofilia resulta una premisa esencial para la subsistencia de las especies arvenses en este cultivo, pero no resulta una limitación. A su vez, el monocultivo constituye una práctica tan recurrente que ha favorecido no solo la persistencia de infestaciones, ya conocidas desde antaño, sino la expansión de otras procedentes de áreas cálidas del continente asiático o americano. La importación de lotes de grano de arroz sin un control de certificación o cuarentena previo, ha constituido la principal vía de introducción de estas especies (Recasens y Conesa, 2003). Podemos citar, como casos más relevantes registrados en las últimas décadas, las especies de los géneros Leptochloa, Leersia y Heteranthera. Todas ellas vienen a mostrar no solo los rasgos propios adaptativos a ambientes de alta humedad, sino también la importante presión de diásporas que tiene lugar dada su alta fecundidad y fácil dispersión.
El género Leptochloa (figura 1) incluye las subespecies Leptochloa fusca subsp. uninervia y L. fusca subsp. fascicularis. Aunque detectadas ya en la década de los años 90 , su presencia se generalizó en todas las zonas arroceras de España a partir del año 2000 , alcanzando una singular importancia en Extremadura y Valencia (Osca, 2013). Ambas subespecies, muestran una fenología singular, pudiendo desarrollar de forma continua nuevos hijuelos a lo largo de todo el periodo vegetativo. Esta plasticidad fenológica garantiza una continua fecundidad. Dentro de cada zona arrocera, las posibilidades de expansión entre campos, se debe a los métodos de riego, la circulación de agua y el desplazamiento de maquinaria. El año 2006 se declaró oficialmente su presencia en el Delta del Ebro (DOGC 4671, de 7 de julio de 2006) (DOGC, 2006a) y desde entonces se llevan a cabo en esa zona tareas para su contención, proceso que ha permitido que ese problema en los arrozales del Delta sea menor que en otras zonas arroceras de España.

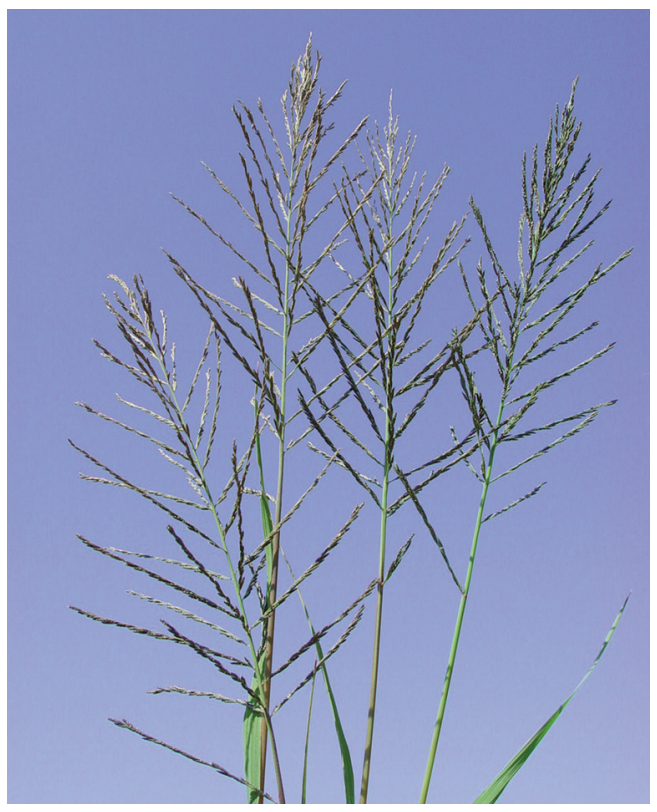

Figura 1. Leptochloa fusca subsp. fascicularis. Figure 1. Leptochloa fusca subsp. fascicularis. 
Otro caso que ha mostrado una rápida expansión en campos de arroz lo constituye Leersia oryzoides. Esta especie es una gramínea plurianual, nativa de zonas templadas y tropicales, y extendida por ambientes húmedos de zonas cálidas de Europa. Durante su ciclo, la planta emite unos rizomas subterráneos que le permiten rebrotar en primavera. La especie fue observada por primera vez en España en los márgenes de campos de arroz de la zona de Pals (Girona) (Vilà, 1997). En 2004 se cita su presencia como especie rara en los arrozales de la Albufera de Valencia (Carretero, 2004). Tras los primeros casos detectados como especie infestante en el Delta del Ebro en 2006, el Servicio de Sanidad Vegetal de la Generalitat de Cataluña establece una Orden declarando oficialmente su presencia (DOGC 4671, del 7 de julio de 2006) (DOGC, 2006a).

Otro caso singular acaecido, en campos de arroz, en la década de los años 90 es la presencia de las especies Heteranthera reniformis y $\mathrm{H}$. limosa. Ambos casos se detectaron inicialmente en Aragón (Zaragoza et al., 1993; Conesa y Sanz-Elorza, 1998). Ambas especies son originarias de zonas húmedas tropicales y subtropicales de América del Sur. Se observó en el Delta del Ebro pocos años después, pero al ser su presencia muy extendida no se incluyó la especie en la Orden antes citada (DOGC 4671, de 7 de julio de 2006) (DOGC, 2006a).

\section{Campos de maíz}

La comercialización e importación de grano de diferentes áreas geográfica y las limitaciones de control en destino han permitido la expansión de ciertas especies infestantes que han devenido invasoras en campos de maíz. El ciclo estival del cultivo, su necesidad de regadío, el incremento de superficies cultivadas e incluso, en algunos casos, el monocultivo, han creado un escenario favorable a la instalación, persistencia y expansión de especies exóticas. La persistencia y estabilidad de es- pecies con unos rasgos funcionales específicos responde precisamente a la selección de especies funcionalmente similares al propio cultivo. Entre estos atributos sobresalen la tolerancia a altos niveles de nitrofilia, metabolismo C4 y altas tasas fotosintéticas (JuárezEscario et al., 2018), a los que podemos añadir una alta fecundidad y persistencia de las semillas en el suelo (Recasens y Conesa, 2003).

No obstante la presencia de especies exóticas en este cultivo no siempre ha devenido en invasiones. En Cataluña se han citado casos de especies como Sida spinosa, Cassia obtusifolia o Sesbania exaltata (Recasens y Conesa, 1995) que, al no haber sido observadas de nuevo, apenas han pasado de una presencia puntual o efímera. Encontramos otro grupo de especies que han mostrado cierto protagonismo a nivel local, como Ipomoea purpurea, Bidens frondosa u Oxalis latifolia, pero su presencia está restringida -por el momento- a unos pocos campos. Esta desigual expansión de unas y otras especies, constituye un paradigma ecológico que debe encontrar explicación en los atributos intrínsecos que definen la adaptabilidad de estas especies a un nuevo hábitat, y en concreto al manejo imperante en el cultivo.

De los distintos casos habidos en campos de maíz de Cataluña, algunos de ellos merecen una especial mención. En la década de los años 80 se detecta la presencia de Abutilon theophrasti (figura 2) en campos de maíz del Pla d'Urgell (Lleida). Su gran expansión se vio favorecida por dos causas principales. Por un lado, su alta tolerancia a la nitrofilia, favoreciendo un gran vigor y unas altas tasas de fecundidad (Recasens et al., 2005). A su vez, la alta persistencia de las semillas en el suelo constituyó una garantía de supervivencia para la especie. Por otro lado, esa expansión por el territorio fue coincidente con la ausencia de materias activas herbicidas que fuesen eficaces para su control. Ese lapso de cerca de 10 años permitió convertir a esa es- 


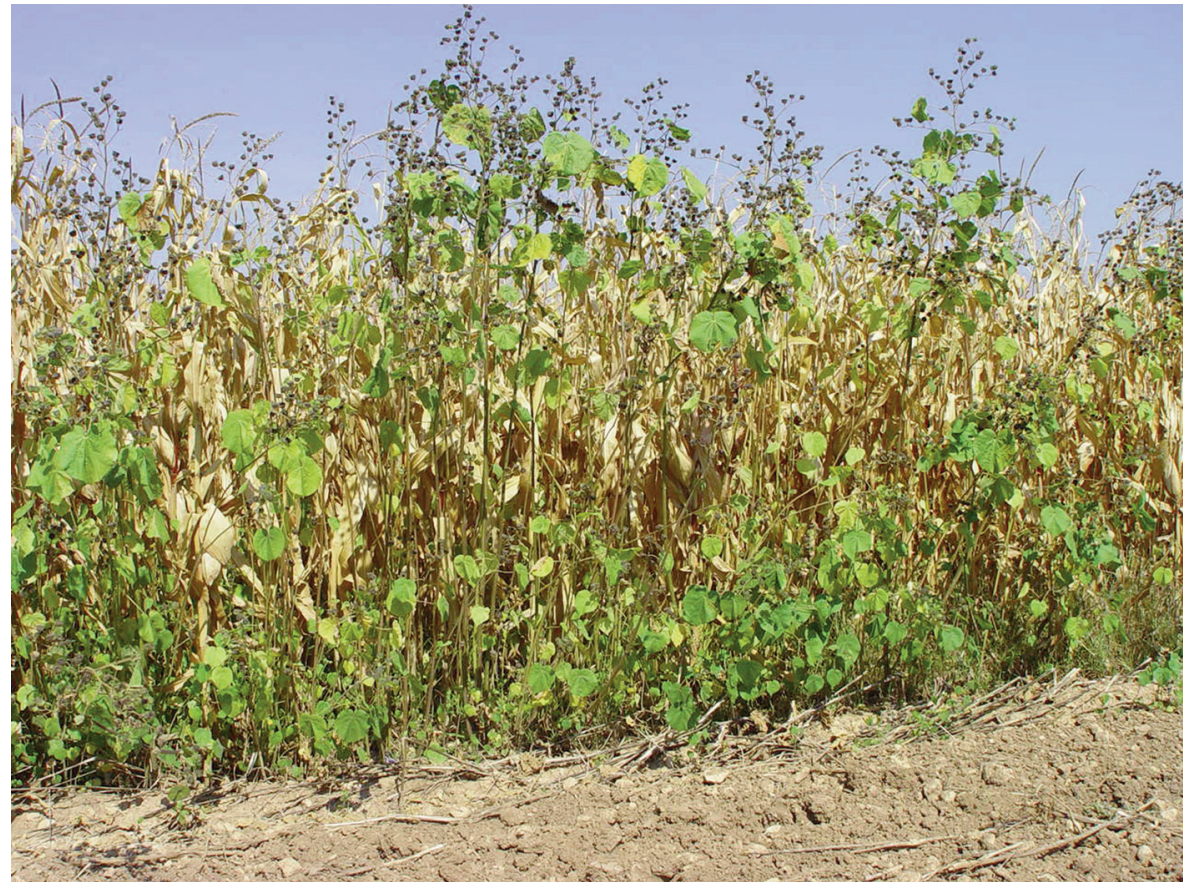

Figura 2. Abutilon theophrasti.

Figure 2. Abutilon theophrasti.

pecie en una de las más problemáticas hoy en día en nuestros campos de maíz. El volumen total de pérdidas causadas por la competencia de esta especie con el maíz, estimado para un periodo de 26 años (1980-2005) en la comarca del Pla d'Urgell (Lleida) es de $4.040 .000 €$, y los costes de llevar a cabo métodos de control químico para ese periodo de tiempo se han estimado en $2.918 .000 €(\operatorname{Re}-$ casens et al., 2007).

La problemática causada por $A$. theophrasti, constituyó un serio y grave precedente. Los siguientes casos detectados en maíz en Cataluña, y que han alcanzado notoriedad, han comportado actuaciones concretas por parte del Servicio de Sanidad Vegetal de Cataluña. Uno de ellos es el protagonizado por la cucurbitácea Sicyos angulatus (figura 3). Esta especie se detectó por primera vez en 2002 como mala hierba en campos de maíz tam- bién del Pla d'Urgell (Lleida), llegando a estar presente, al cabo de dos años, en diez explotaciones distintas. Ante los precedentes sobre la nocividad de esta especie en otros países y el hecho de estar incluida en la Lista de Alerta de la EPPO en 2005, se establecieron protocolos para su contención (Taberner y Sans, 2005). En 2005 se publica una Orden a nivel autonómico (DOGC núm 4315, de 3 febrero 2005) (DOGC, 2005) por la que se declara la presencia de Sicyos angulatus y se establecen las medidas de lucha contra ella. A los 10 años de inicio del programa de erradicación, la especie se encontraba confinada en un solo campo y con una presencia reducida a menos de diez ejemplares.

Otro de los casos registrados en campos de maíz de Cataluña y Aragón, a partir del año 2014, ha sido el "teosinte" (figura 4). Con este término se denominan aquellos ejempla- 


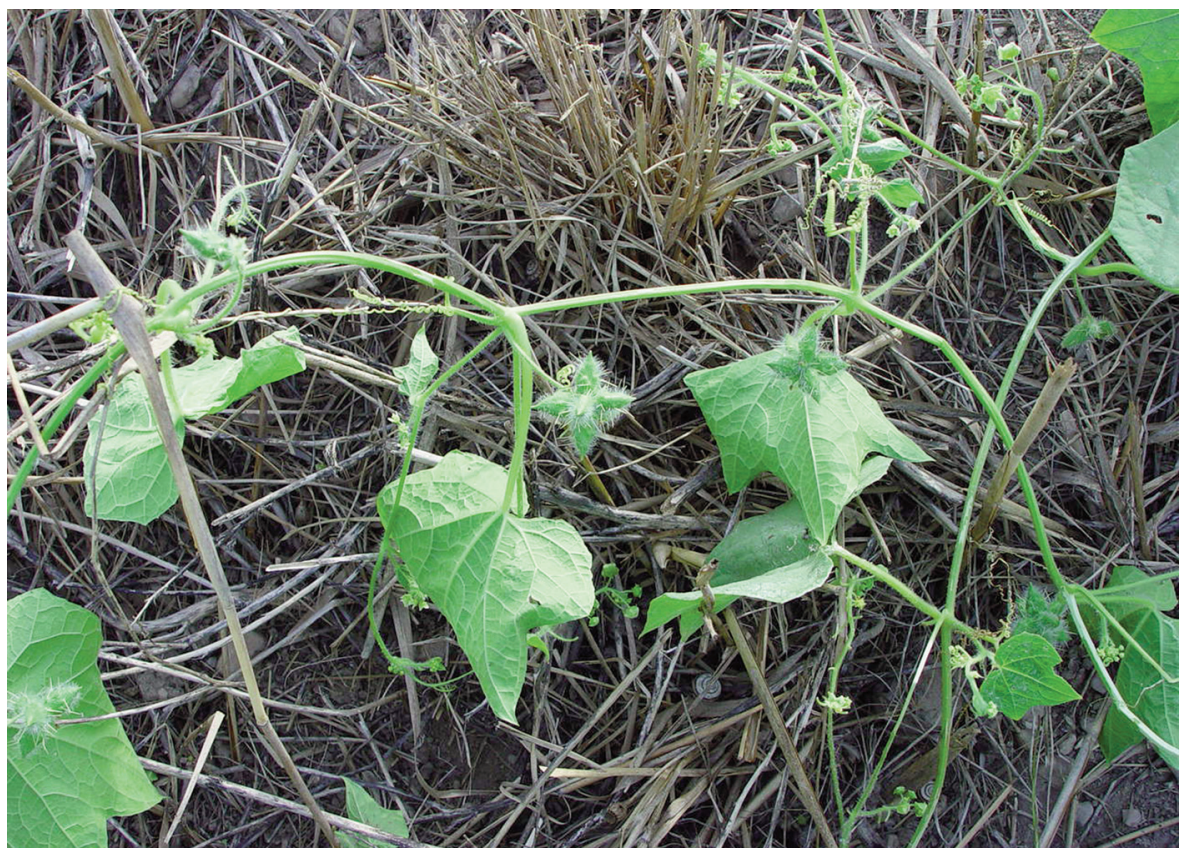

Figura 3. Sicyos angulatus.

Figure 3. Sicyos angulatus.

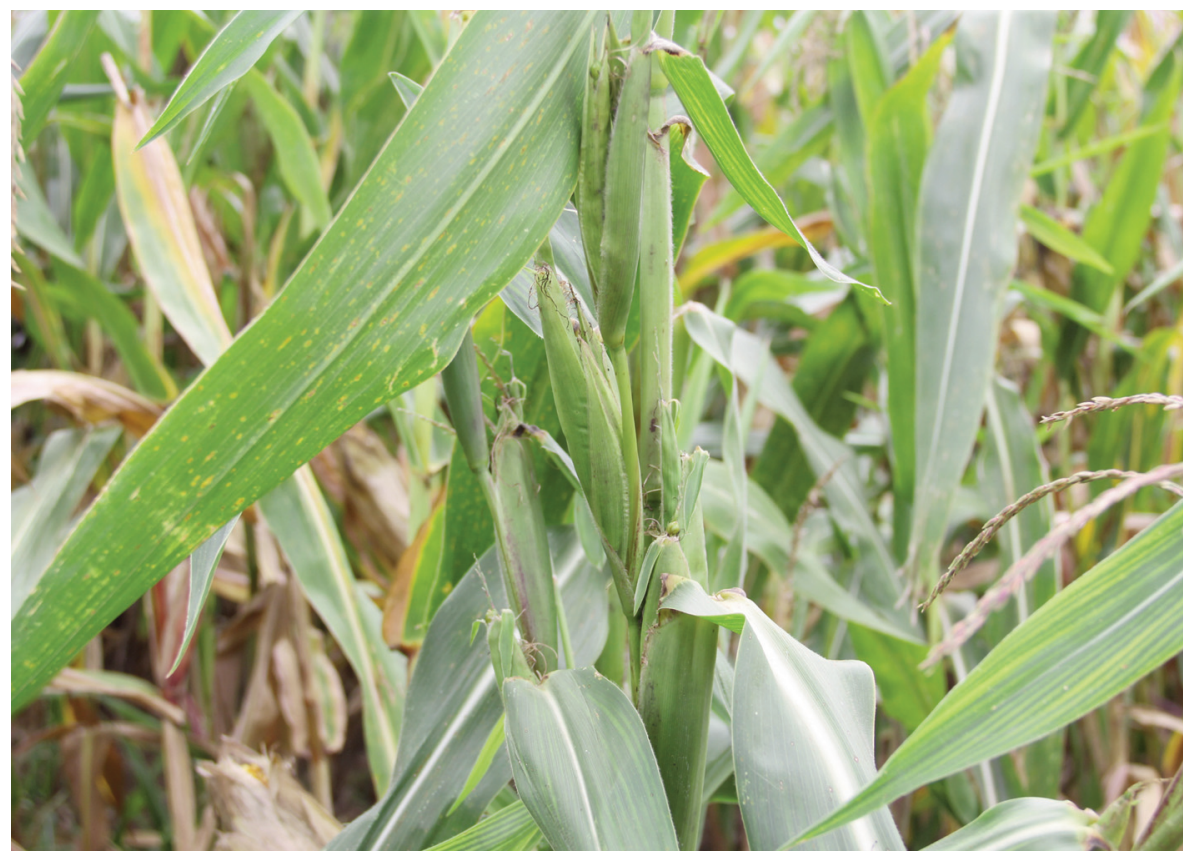

Figura 4. Ejemplares de "teosinte". Figure 4. "Teosinte" specimens. 
res observados y que muestran características híbridas entre el maíz y su ancestro, el auténtico teosinte (Zea mays subsp. parviglumis y Zea mays subsp. mexicana) constituyendo, parece ser, un grupo genético distinto (Díaz et al., 2019). El auténtico teosinte apareció hace 8.000 años y de él derivan los cultivares actuales del maíz después de miles de años de selección. Los ejemplares observados en el Valle del Ebro corresponden a plantas anuales de mayor altura que el propio maíz. Su principal característica vegetativa es su tallo ramificado, que puede producir numerosas mazorcas en cada ramificación. Estas mazorcas son muy delgadas y ramificadas, con un grano de aspecto muy distinto al del maíz. La relevancia de las infestaciones de este "teosinte" trasciende a la problemática de su control; al tratarse de una planta emparentada con el maíz se pueden producir híbridos naturales con éste y originar una alta heterogeneidad en la descendencia (Pardo et al., 2015; Díaz et al., 2019). Los Servicios de Sanidad Vegetal de Aragón y Cataluña Ilevan varias campañas tomando serias medidas fitosanitarias cautelares de obligado cumplimiento. Tras cinco años después de su detección, las observaciones de campo parecen indicar que la especie va en regresión. Este no es un hecho casual. La diferencia respecto a otros casos citados anteriormente se debe a la nula posibilidad de la población de "teosinte" de poder naturalizarse en ambientes ruderales, fuera de los campos de maíz. Su alta dependencia del manejo que se establece en el campo y su relativa corta supervivencia del banco de semillas ha permitido confinar el problema en un cultivo concreto y en un área geográfica también concreta.

Desde hace pocos años, un nuevo caso problemático se ha registrado en campos de maíz de Cataluña y en la parte oriental de Aragón. Se trata de Amaranthus palmeri (figura 5), especie originaria del extremo sur-occidental de Estados Unidos, donde infesta campos de algodón, maíz y soja. $A$. palmeri es una plan- ta dioica, que puede alcanzar entre 1,5 y 2,5 metros y muestra unas largas inflorescencias terminales que acaban arqueándose por su peso. Los pies femeninos, pueden llegar a mostrar una fecundidad superior a las 250.000 semillas por planta (Sellers et al., 2003) y con una viabilidad de hasta 12 años. En 2007 se detectó una población estable en los márgenes de un campo de maíz y en ambientes ruderales y viarios próximos a la ciudad de Lleida (Recasens y Conesa, 2011). En 2017 se encontraron varios campos de maíz de Lleida y Huesca infestados por esta especie (Recasens et al., 2017). A este problema hay que añadir su potencial capacidad de desarrollar biotipos resistentes a diferentes grupos de herbicidas. A nivel europeo, y desde 2014, está incluida dentro de la EPPO Alert List (EPPO, 2019). Su vigorosidad, alta fecundidad y rápida expansión definen un nuevo y alarmante caso de mala hierba invasora. Los Servicios de Sanidad Vegetal de Cataluña y Aragón están adecuando medidas para su contención. Precisamente, de forma reciente, se ha publicado una Orden a nivel autonómico (DOGC núm 7959, de 13 septiembre 2019) (DOGC, 2019) por la que se declara la existencia de Amaranthus palmeri y se califica de utilidad pública la lucha contra ella.

\section{Campos de alfalfa}

El riego y la recurrencia de la siega a lo largo de la campaña condicionan el hábito y la adaptabilidad de las especies infestantes en campos de alfalfa. La capacidad de rebrote mediante la formación de nuevos hijuelos -como en el caso de gramíneas- o la regeneración de la planta partir de yemas basales tras la fragmentación del tallo -caso de hemicriptófitos- resulta una estrategia exitosa. Como ejemplos de gramíneas podemos citar dos especies, Panicum dichotomiflorum (figura 6) y Eleusine indica (figura 7), que aun siendo de ciclo anual, pueden adaptarse con éxito al manejo imperante en el cultivo dada 


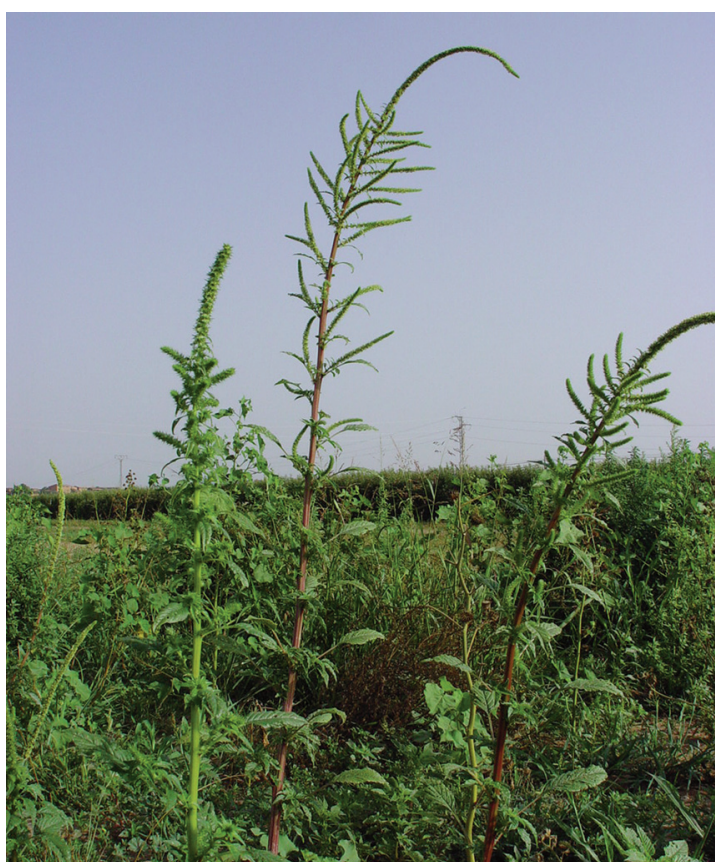

Figura 5. Amaranthus palmeri. Figure 5. Amaranthus palmeri.

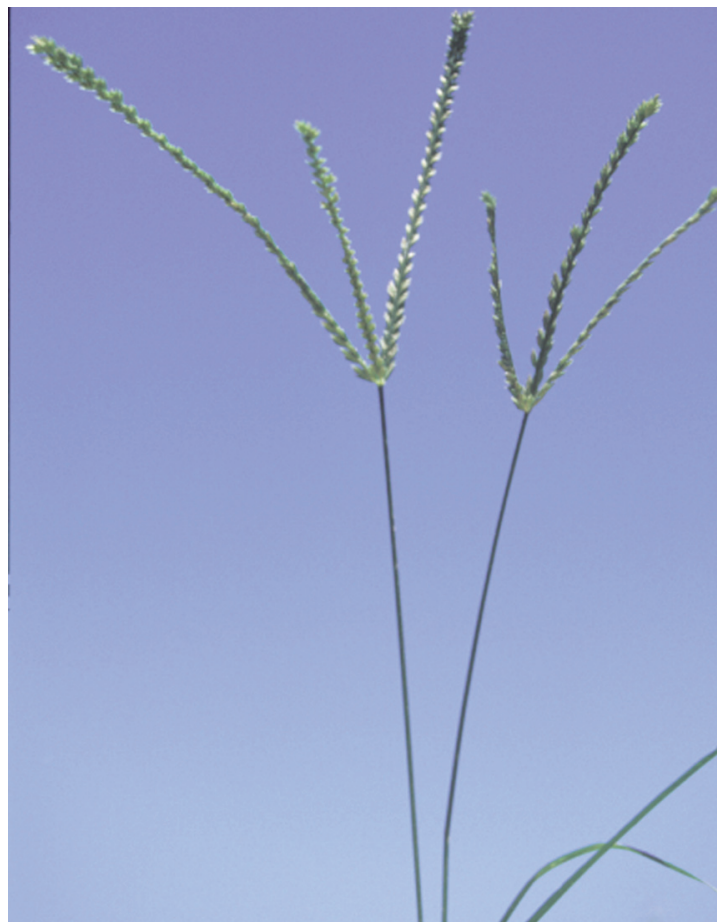

Figura 7. Eleusine indica. Figure 7. Eleusine indica.

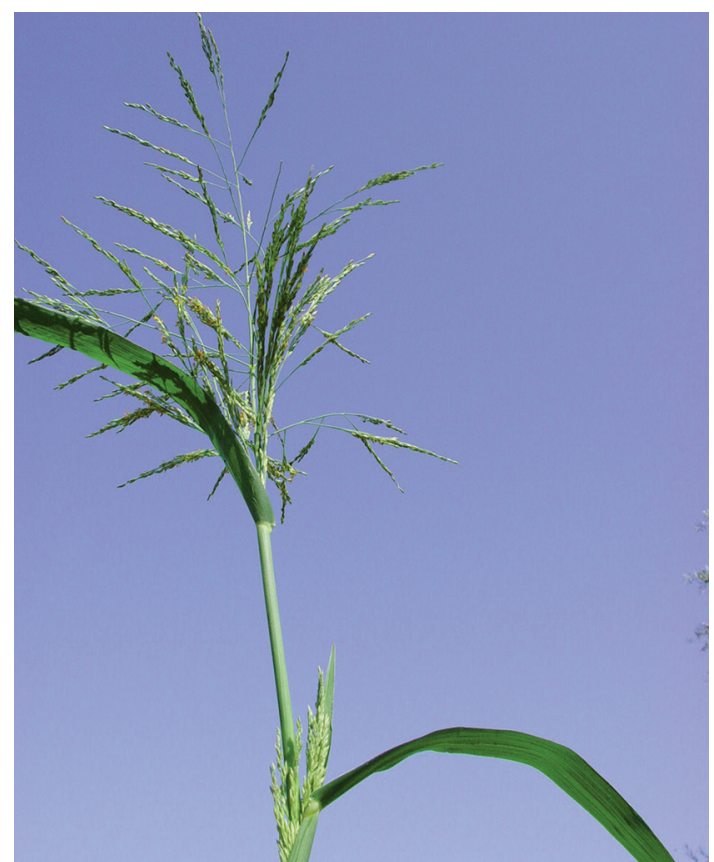

Figura 6. Panicum dichotomiflorum. Figure 6. Panicum dichotomiflorum.

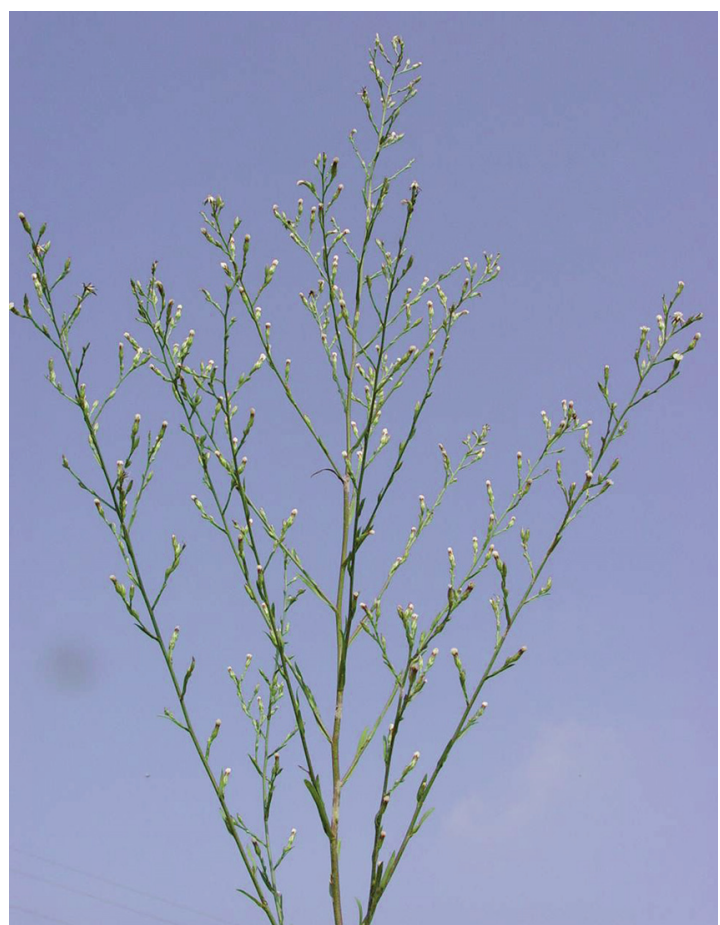

Figura 8. Aster squamatus. Figure 8. Aster squamatus. 
su alta capacidad de regeneración de hijuelos a partir de yemas intravaginales. Ambas especies muestran metabolismo C4, por lo que su presencia está restringida al periodo comprendido entre junio y octubre. Su adaptación a éste y otros cultivos de regadío, les permite mostrar diferente arquitectura vegetativa. Ante un manejo definido por siegas frecuentes muestran un recurrente y activo desarrollo de hijuelos que alcanzan la floración en pocos días, garantizando la producción de semillas de forma continua. Ambas especies aparecen también en frutales de regadío, donde suele ser recurrente el pase de picadora por las calles.

Otro caso singular lo constituye Aster squamatus (figura 8) una asterácea de origen americano de gran porte que dada su capacidad de regenerar yemas a partir de la parte basal del tallo muestra una alta adaptación a sistemas agrícolas con perturbación por segadora (p.e. campos de alfalfa) o mediante picadora (p.e. frutales o viñedos). El hándicap de su presencia lo constituye la lignificación de la base del tallo a lo largo de los años, que no solo resulta persistente sino además muy vigorosa, facilitando la regeneración de nuevas yemas $y$, por ende, nuevas ramificaciones. Además, aquellos ejemplares que llegan a florecer, permiten dispersar sus pequeños aquenios mediante procesos de anemocoria, favoreciendo nuevas germinaciones en un sistema donde los recursos por agua y nutrientes no son limitantes. Estando ya la planta desarrollada su sensibilidad a los posibles tratamientos herbicidas es prácticamente nula, y más aún si la planta ha lignificado su base al llevar dos o más años instalada.

\section{Cultivos leñosos de regadío}

En los campos de frutales de regadío el distinto manejo que se lleva a cabo permite albergar unas $u$ otras especies de malas hierbas. Uno de los principales factores lo constituye el tipo de riego. Tanto en campos con riego por inundación como por riego por goteo, el manejo de la vegetación bajo la línea del cultivo suele realizarse mediante la aplicación de herbicidas. Este hecho ha constituido, en las últimas décadas, un cambio significativo en el manejo de la vegetación. Tradicionalmente, el manejo se realizaba mediante segadoras de discos tanto en la calle como bajo la línea; en la actualidad se ha generalizado el pase de picadora, permitiendo un corte más bajo y eficaz, combinado con aplicaciones de herbicidas bajo la línea del cultivo. Precisamente, la frecuencia del pase de picadora define el tipo de intervención mecánica y la vegetación que allí se instala. En aquellos casos donde existe riego por inundación se favorecen unas condiciones de alta humedad, que junto al tipo manejo de la cubierta, permiten albergar especies de hábito anual o de tipo hemicriptófito capaces de regenerarse a partir de yemas ubicadas a nivel del suelo. Ciertas gramíneas con dichos atributos biológicos han encontrado, en las últimas décadas, un escenario adecuado para su instalación y persistencia. Como ejemplos de anuales citamos $P$. dichotomiflorum y $E$. indica; como ejemplos de hemicriptófitos destacan: Bromus catharticus, Paspalum dilatatum y Sporobolus indicus. Las tres con distinto origen: América del Sur, América del Norte y Sur de Asia, respectivamente. Se desconoce las causas de su introducción, pero en el caso de las dos primeras parecen tratarse de especies introducidas como cultivos forrajeros o para su uso como cubierta vegetal. $P$. dilatatum se cultiva también como césped ornamental.

Las aplicaciones herbicidas realizadas bajo la línea del cultivo, especialmente en campos con riego por goteo, ha creado un nuevo escenario para la vegetación herbácea. La diferente sensibilidad de la flora allí presente a los herbicidas que suelen aplicarse, especialmente glifosato, ha favorecido la selección de ciertas especies en detrimento de otras. Uno de los casos más relevantes lo constituyen Conyza bonariensis (figura 9). Esta especie, originaria de América del Sur, es un claro ejemplo de cambio en su estrategia evolutiva 


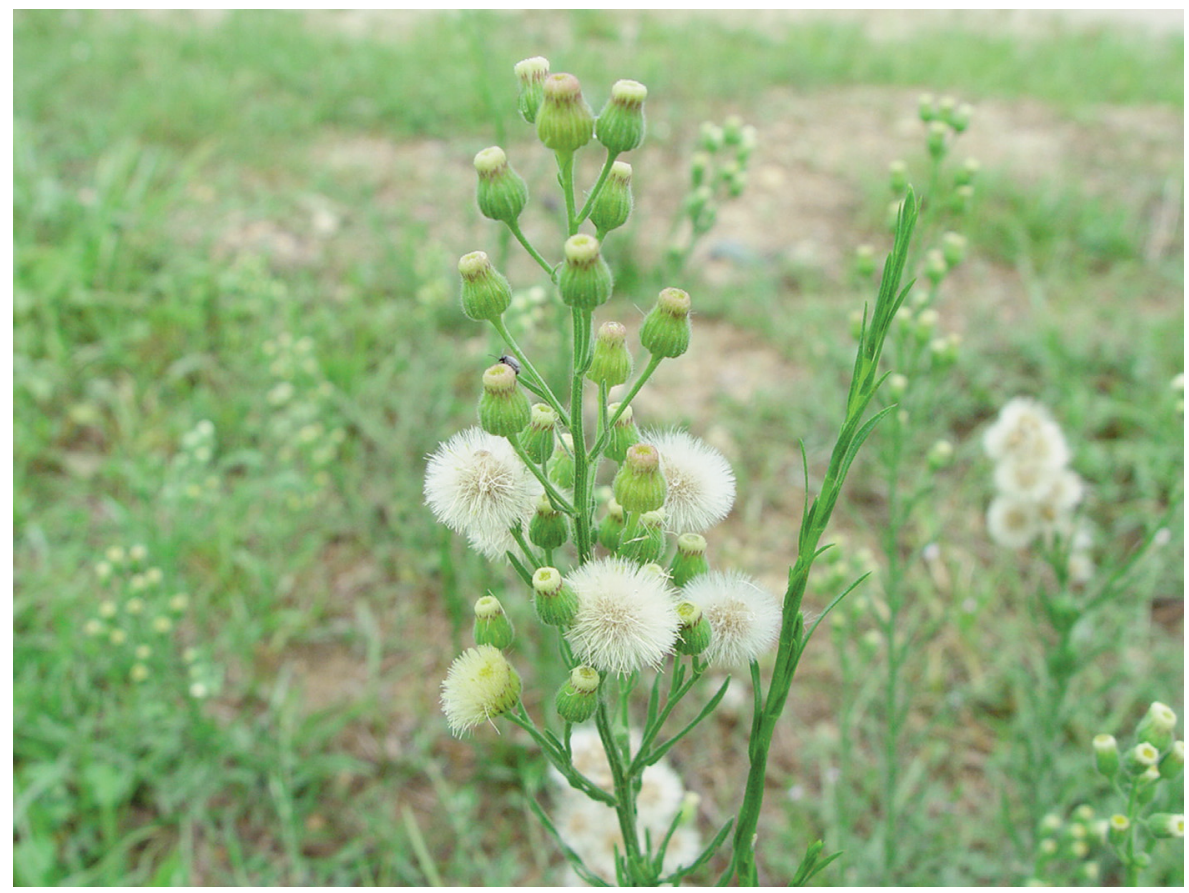

Figura 9. Conyza bonariensis.

Figure 9. Conyza bonariensis.

como planta invasora. Su expansión por sistemas agrícolas y ambientes ruderales ha tenido lugar varias décadas después de su introducción (Recasens y Conesa, 2003). Es a partir de los años 90 cuando su presencia se ha visto generalizada. La continua y exitosa dispersión de aquenios por el viento garantiza la expansión de la población, pudiendo instalarse en sistemas donde no hay laboreo del suelo y facilitar su germinación superficial. Los espacios bajo la línea de frutales o viñedos, que suelen ser tratados con herbicidas, son escenarios muy favorables a acoger la presencia de estas especies. C. bonariensis muestra tanto emergencias otoñales como de primavera, y en cada estación dentro de un amplio gradiente de semanas (especialmente entre marzo y mayo). Este escalonamiento de las emergencias permite esquivar el efecto de las aplicaciones herbicidas. Por otra parte, una vez que la planta alcanza el tamaño de roseta disminuye mucho su sensibilidad y las aplicaciones resultan de muy baja eficacia. Por último, la presión de selección impuesta por la reiteración en el uso de un mismo tipo de herbicida ha conllevado la aparición de biotipos resistentes. Se conocen, para C. bonariensis, casos de resistencia a glifosato en frutales en Aragón (Langa et al., 2015).

\section{Entorno legislativo}

La gestión de las especies exóticas en Cataluña se halla regulada a tres niveles legislativos: el comunitario, el estatal y el autonómico.

A nivel comunitario el documento normativo es el Reglamento 1143/2014 del Parlamento Europeo y del Consejo, de 22 de octubre de 
2014 (DOUE, 2014) sobre la prevención y la gestión de la introducción y propagación de especies exóticas invasoras. Este reglamento permite, mediante actos de ejecución, elaborar listas de especies exóticas actualizadas que son preocupantes para la Unión Europea. Hasta el momento son dos las publicadas: reglamentos de ejecución 2016/1141, de 13 de julio, y 2017/1263, de 12 de julio (DOUE, 2016; DOUE, 2017). A nivel estatal la gestión de las especies exóticas invasoras está mediada por el Real Decreto 630/2013, de 2 de agosto (BOE, 2013), que regula el Catálogo Español de Especies Exóticas Invasoras (Ley 42/2007 de Patrimonio Natural y de la Biodiversidad) (BOE, 2007), junto con la Sentencia de 16 de marzo de 2016 del Tribunal Supremo, que anula algunos extremos del Real Decreto anterior (BOE, 2016). Ambas normativas establecen listados de especies invasoras en base a criterios diferentes, siendo la norma estatal la que recoge un mayor número de ellas.

Desde el punto de vista legislativo, tanto a nivel europeo como nacional, las invasiones producidas por especies vegetales en los sistemas agrícolas han tenido una consideración desigual respecto a las protagonizadas por plagas de insectos o patógenos. Dentro de la Directiva Europea 2000/29/CE (DOCE, 2000) para la prevención de la introducción de organismos nocivos para los vegetales o productos vegetales, se incluía un largo listado de insectos, ácaros, nematodos, hongos y virus. Tan solo incorporaba el género vegetal Arceuthobium, para las especies no europeas, aunque lo era por su carácter parásito. Ninguna especie vegetal con aptitud como mala hierba se incluía en ese listado de organismos nocivos para los cultivos.

Por otra parte, el catálogo español de especies exóticas invasoras (BOE, 2013), elaborado a partir de las bases establecidas en el derogado Real Decreto 1628/2011 de 12 de diciembre (BOE, 2011), en su preámbulo especificaba:
La introducción de especies invasoras puede ocasionar graves perjuicios a la economía, especialmente a la producción agrícola, ganadera y forestal, e incluso a la salud pública. Sin embargo, la inclusión de los sistemas agrícolas como escenarios susceptibles a la invasión, no se ha visto reflejada en la definición de especie exótica invasora que se da más adelante en ese mismo decreto: Especie exótica es aquella que se introduce o establece en un ecosistema o hábitat natural o seminatural, y que es un agente de cambio y amenaza para la diversidad biológica nativa, ya sea por su comportamiento invasor, o por el riesgo de contaminación genética. A su vez, si miramos la lista de especies vegetales incluidas como invasoras en el Real Decreto de 630/2013 (BOE, 2013) la gran mayoría de ellas corresponden a especies arbóreas y arbustivas presentes en sistemas naturales, y el área de prevención de su presencia, corresponde en la mayoría de casos, a territorios insulares (Baleares y Canarias). Resulta por tanto paradigmático que se exponga el riesgo para la agricultura de la presencia de especies invasoras y no se incluya ninguna especie de mala hierba exótica que muestre, o haya mostrado, riesgo de invasión.

Podríamos pensar que estas especies están recogidas en la Ley 43/2002 de Sanidad Vegetal (BOE, 2002) y que por lo tanto es ésta y no aquélla la norma legal que regula su presencia. La ley de Sanidad Vegetal creó un marco legal apropiado para la defensa de vegetales y de productos vegetales contra los daños producidos por plagas. Hace mención a la necesidad de evitar la introducción de organismos de cuarentena y a otros aspectos relacionados con la defensa fitosanitaria. Sin embargo, tampoco hace una mención expresa a la tipología de organismos ni ha sido desarrollada de forma detallada por ninguna normativa que incluya a las malas hierbas exóticas invasoras. Es evidente que nos encontramos ante un enfoque planteado más 
como un problema medioambiental que agronómico. Ello ha llevado, a una situación de cierto vacío legislativo en materia de prevención y de actuación frente a la presencia de especies vegetales alóctonas que han mostrado una clara expansión como malas hierbas en nuestros cultivos.

En Cataluña, además de aquellos marcos normativos comunitario y estatal es también de aplicación la normativa autonómica propia que se concreta en el Decreto 137/2014, de 7 de octubre (DOGC, 2014) sobre medidas para evitar la introducción y propagación de organismos nocivos especialmente peligrosos para los vegetales y productos vegetales, que deroga uno anterior (Decreto 6/1985, de 14 de enero) (DOGC, 1985). Este Decreto 137/2014, no es solo el instrumento jurídico para declarar oficialmente un organismo nocivo, sino que además es la vía para dar a conocer nuevos focos expansivos de especies nocivas y las zonas demarcadas dentro de las cuales es preceptivo aplicar las medidas que se dicten. El Decreto también define los organismos nocivos para los vegetales como aquellos: a) de cualquier especie que sea identificada por la normativa europea; b) se hallen incluidos en alguna de las decisiones comunitarias sobre medidas de emergencia y/o control; c) aquellos que se hallen determinados en las listas de alerta de la Organización Europa y Mediterránea para la Protección de las Plantas (OEPPEPPO), y d) todos aquellos otros que, siendo detectados en el territorio catalán por el departamento competente en materia de sanidad vegetal y con una base científica sólida, dé lugar a la adopción de medidas cautelares para evitar que pueda producir perjuicios económicos o medioambientales. El órgano competente es actualmente la Direcció General d'Agricultura i Ramaderia.

\section{Listas de alerta y catálogos de especies exóticas}

En Cataluña además de los textos normativos y como complemento en la detección de especies exóticas y la toma de decisiones, existen y se usan documentos científico-técnicos que aportan catálogos o listados de especies exóticas con valoraciones en base a su naturalización y agresividad.

En este sentido, la Organización Europea y Mediterránea para la Protección de las Plantas-EPPO- (EPPO, 2019) tiene sus listas A1 (organismos de cuarentena no presentes en el ámbito de la EPPO) y A2 (aquellos presentes en alguna zona del territorio) que se actualizan frecuentemente y que conviene conocer. Las listas A1 y A2 de la EPPO tienen carácter consultivo, por lo que no todos los organismos incluidos en ellas están considerados oficialmente como de cuarentena en la Directiva 2000/29/CE (DOCE, 2000). Pero también posee la lista de especies invasoras y la lista de alerta; esta última consiste en una breve relación de especies exóticas que representan un riesgo para los estados miembros de la EPPO y que además de generar una alerta preventiva puedan ser utilizadas para establecer una evaluación de análisis de riesgos. Esta lista de alerta no es, de ningún modo, una lista de cuarentena, pero es la que recoge el Decreto 137/2014 catalán (DOGC, 2014) como referencia para poder declarar oficialmente una planta como organismo nocivo.

Por otro lado existe también el Sistema de Información de Especies Exóticas de Cataluña. Se trata de una base de datos sobre especies exóticas en Cataluña que identifica y caracteriza todas las especies alóctonas de distintos grupos de organismos. Forma parte del proyecto EXOCAT (EXOCAT, 2019) en el que colaboran El Centre de Recerca Ecològica $i$ Aplicacions Forestals (CREAF) y el Servei de Biodiversitat i Protecció dels Animals de la Generalitat de Catalunya. Esta base de datos 
reúne la mayoría de la información sobre especies exóticas en cuanto a la distribución, procedencia, vía de entrada y estado de invasión o estatus en el territorio catalán. Para las especies vegetales, algunas de esas citas son antiguas y corresponden a especies que no han logrado establecerse, pero las hay que corresponden a introducciones muy recientes que es necesario monitorizar para evitar su establecimiento, expansión y peligrosidad. El documento también diferencia las especies translocadas, es decir especies autóctonas de algunas áreas de la Península Ibérica pero que han sido introducidas en territorios donde previamente no existían. Estas especies pueden también provocar impactos de carácter similar a los que generan las especies exóticas en sentido amplio.

A través del Decreto 6/1985 (DOGC, 1985), hoy derogado a partir del Decreto 137/2014 actualmente vigente (DOGC 2014), y como ya se ha mencionado más arriba, se publicaron en los años 2005 y 2006 diversas órdenes (DOGC, 2005,2006 a y 2006b) mediante las cuales se declararon oficialmente organismos nocivos -malas hierbas- en Cataluña a las especies Sicyos angulatus, Leptochloa sp., Leersia oryzoides, Sagittaria sp. y Solanum carolinense. Las declaraciones también establecían las medidas obligatorias de lucha. Para Amaranthus palmeri, la orden se ha publicado de forma reciente (DOGC, 2019).

\section{Análisis crítico de la inclusión de especies arvenses en listas de nocividad}

El amplio conjunto de datos generados a partir de las listas oficiales de especies exóticas invasoras y de las listas referentes a especies con potencial invasor y de peligrosidad todavía desconocida, obliga a plantearnos: ¿hasta qué punto las listas oficiales recogen todas las especies exóticas con potencial nocivo para los cultivos? Como respuesta, ex- ponemos una visión crítica al respecto comparando la información obtenida a partir de nuestras observaciones con la información aportada en esas listas.

En la tabla 1 se presentan las especies de malas hierbas exóticas que hallándose en Cataluña son consideradas nocivas o potencialmente nocivas. Estas especies se hallan reunidas en: a) listas de especies exóticas invasoras preocupantes para la Unión Europea (Reglamento UE 1143/2014) (DOUE, 2014); b) especies incluidas a nivel nacional en el RD 630/ 2013) (BOE 2013); c) especies recogidas, a nivel de Cataluña, en el DOGC (DOGC, 2005, 2006a y 2019) a través de órdenes de declaración oficial de nocivas; d) especies incluidas en las listas de la EPPO (EPPO, 2019), y e) especies incluidas en la lista EXOCAT (EXOCAT, 2019).

Las especies que fueron declaradas oficialmente malas hierbas nocivas en Cataluña en los años 2005 y 2006 (DOGC, 2005; 2006a y 2006b), no han desarrollado un grado de amenaza para los cultivos, a excepción de las especies de Leptochloa, que si bien son bastante frecuentes en los campos de arroz del Delta del Ebro, su nocividad es menor que la que se ha detectado en otras zonas arroceras de España. Asimismo, las medidas desarrolladas sobre Sicyos angulatus en los cultivos de maíz (Taberner y Sans, 2005) han permitido evitar no sólo su expansión sino incluso establecer una eficaz contención. En este sentido debe destacarse la celeridad con la que actuó la Administración al divulgar la potencial amenaza de esas especies al poco tiempo de conocerse la introducción, así como el monitoreo posterior que se ha prolongado más de diez años.

Por su parte Amaranthus palmeri viene recogido en la lista de Alerta de EPPO (EPPO, 2019) y en EXOCAT (EXOCAT, 2019) por su seria amenaza. Su presencia cada vez más frecuente y abundante, requiere una declaración administrativa como mala hierba invasora con el fin de promover su contención y evitar su ex- 
Tabla 1. Especies vegetales, presentes en sistemas agrícolas de Cataluña, consideradas nocivas por su potencial invasor, según diferentes fuentes: Reglamento Unión Europea (DOUE, 2014); Catálogo Español (BOE, 2013); DOGC (2005, 2006a, 2006b, 2019), lista de alerta EPPO (2019) y lista EXOCAT (2019).

Table1. Plant species, present in agricultural systems of Catalonia, considered harmful due to their invasive potential, according different sources: European Union Law (DOUE, 2014); Official State Gazette (BOE, 2013); DOGC (2005, 2006a, 2006b, 2019), EPPO Alert List (2019) and EXOCAT list (2019).

\begin{tabular}{|c|c|c|c|c|c|}
\hline & DOUE & $\mathrm{BOE}$ & DOGC & EPPO & EXOCAT \\
\hline Amaranthus palmeri & . & . & $x$ & $X^{A}$ & $X^{e s}$ \\
\hline Ambrosia artemisiifolia & . & $x$ & . & $\mathrm{X}^{\prime}$ & $X^{\text {no es }}$ \\
\hline Araujia sericifera & . & $x$ & . & $X^{\prime}$ & $x$ \\
\hline Azolla filiculoides & . & . & $x$ & $x^{\prime}$ & $x$ \\
\hline Bidens frondosa & . & . & . & $X^{\prime}$ & $x$ \\
\hline Bidens subalternans & . & . & . & $X^{A}$ & $x$ \\
\hline Eichhornia crassipes & $x$ & $x$ & . & $X^{A 12}$ & $X^{e s}$ \\
\hline Leersia oryzoides & . & . & $x$ & . & . \\
\hline Leptochloa sp. ${ }^{1}$ & . & . & $x$ & . & $x^{e s}$ \\
\hline Sagittaria sp. ${ }^{2}$ & . & . & $x$ & . & $\mathrm{X}^{\text {no es }}$ \\
\hline Oxalis pes-caprae & . & $x$ & . & $\mathrm{X}^{\prime}$ & $x$ \\
\hline Paspalum distichum & . & . & . & $X^{\prime}$ & $x$ \\
\hline Sicyos angulatus & . & . & $x$ & $\mathrm{X}^{\prime}$ & $X^{i}$ \\
\hline Senecio inaequidens & . & $x$ & . & $x^{\prime}$ & $x$ \\
\hline Solanum carolinense & . & . & $x$ & . & . \\
\hline
\end{tabular}

${ }^{1}$ EXOCAT distingue: L. fusca subsp. uninervia y L. fusca subsp. fascicularis.

2 Solo para especies de Sagittaria no europeas (S. montevidensis subsp. calycina).

A: en lista de alerta; A12: en listas A1/A2; I: en lista de plantas invasoras.

es: especie establecida; no es: especie no establecida; $i$ : introducida.

pansión. Los Servicios de Sanidad Vegetal de Cataluña y Aragón han iniciado actuaciones en ese sentido, habiéndose publicado, de forma reciente, la correspondiente Orden en Cataluña (DOGC, 2019). Por su parte la presencia de Bidens subalternans siendo más frecuente en sistemas naturales -especialmente en sistemas riparios- que en sistemas agrícolas, debería ser motivo también de vigilancia.
Sin embargo, desde el punto de vista crítico cabe también resaltar la escasa o nula transferencia de datos entre los responsables de EXOCAT y la Conselleria de Agricultura y viceversa, puesto que se detecta que: a) especies exóticas que han sido declaradas nocivas no constan en los listados de EXOCAT o se incorporan tarde (casos de Solanum carolinense, Leersia oryzoides, Leptochloa fusca subsp. fas- 
cicularis); b) algunos de los géneros declarados como nocivos por parte de la Administración contienen especies que no deben ser consideradas como exóticas (Sagittaria sp.) y c) existe una cierta tendencia a subestimar los estatus de algunas especies que, al hallarse poco representadas en el medio natural (Leersia oryzoides), EXOCAT no las recoge o no las contempla como amenaza potencial como malas hierbas de cultivos.

De modo similar actúa el Servei de Biodiversitat i Protecció dels Animals de la Generalitat de Catalunya que subestima las especies exóticas recientemente introducidas en hábitats ruderales y campos de cultivo al no considerarlas de su competencia. En base a este criterio, no se establecen protocolos para su prevención y lucha por lo que se pierde eficacia para su erradicación.

\section{Situación actual en sistemas agrícolas en Cataluña. Evaluación de riesgos}

En el punto 4 se han expuestos distintos casos de invasiones vegetales registradas en los sistemas agrícolas de Cataluña en las últimas décadas. La desigual expansión e impacto generado según especies, responde a la posibilidad, o no, de una rápida actuación y establecimiento de estrategias de contención. Un buen paradigma son los casos de Abutilon theophrasti y Sicyos angulatus, iniciados ambos en un mismo tipo de cultivo y territorio, que han devenido en casos muy dispares. La toma de medidas cautelares en 2004 contra S. angulatus ha resultado decisiva. Desconocemos si una decisión similar en 1980 hubiese tenido igual repercusión contra $A$. theophrasti. No obstante, sí podemos afirmar que, aunque la erradicación o contención de una especie con potencial invasor no llegue a resultar del todo exitosa, siempre resultará favorable si se mantienen las medidas cautelares adecuadas. Un ejemplo es la dis- tinta situación de las infestaciones de los campos de arroz del Delta del Ebro respecto a otras zonas arroceras de España por parte de especies del género Leptochloa y Leersia.

Precisamente, en esta dirección se han desarrollado múltiples estrategias basadas en esquemas predictivos de riesgo y de impacto (conocidos como Weed Risk Assessment Tools). El objetivo de dichos esquemas es determinar la probabilidad de entrada, establecimiento, dispersión e impactos potenciales de una determinada especie exótica en una región. Estos cálculos se suelen realizar mediante modelos estadísticos, por medio de la creación de rangos semicuantitativos o mediante puntuaciones cualitativas llevadas a cabo por expertos. La finalidad de estos modelos consiste en caracterizar el potencial invasor de una especie de cara a desarrollar medidas de contención o control y minimizar los daños potenciales que pudiese ocasionar. En la actualidad, en Europa, dichas evaluaciones se llevan a cabo a través de distintos protocolos, incluyendo, entre otros, algunos tan extendidos como EPPO-EIA (Kenis et al., 2012), Harmonia+ (D'hondt et al., 2015), GABLIS (Essl et al., 2011) o GISS (Nentwig et al., 2016). Todos ellos deben cumplir unos estándares mínimos de calidad y seguir la regulación europea (Regulation EU No 1143/2014) (DOUE, 2014).

El desarrollo y aplicación de estos esquemas se ha utilizado en la predicción de invasiones a nivel regional, centrándose de manera preferente en ambientes naturales (K ivánek y Pyšek, 2006; Gassó et al., 2009), si bien hay también ejemplos de su aplicación en ambientes agrícolas, y de manera especial en agroecosistemas mediterráneos (Crosti et al., 2010). Dichos esquemas son particularmente útiles en la evaluación y control de especies fáciles de detectar y que permanecen largo tiempo en fase de naturalización antes de desarrollar un comportamiento invasor (Hulme, 2012). La capacidad predictiva de los diferentes protocolos suele ser elevada cuando 
se aplican de forma independiente $y$, de hecho, su aplicación se ha demostrado eficaz a la hora de reducir daños económicos (Keller et al., 2007). Sin embargo, estudios recientes alertan sobre la subjetividad implícita en las valoraciones de dichos protocolos (Dana et al., 2019) al depender del grado de experiencia del evaluador y de la escala espacial de aplicación, lo que puede dar lugar a inconsistencias en las valoraciones de riesgo cuando se analizan, en conjunto, los resultados de diferentes esquemas (González-Moreno et al., 2019). Por otro lado, la limitación en la funcionalidad de dicha metodología también recae en la dificultad en cuantificar objetivamente los daños causados por las especies evaluadas, en medir la variabilidad y la estocasticidad inherente de los hábitats hospedadores (Hulme, 2012) y en la falta de estandarización de los diferentes protocolos de evaluación diseñados hasta ahora (González-Moreno et al., 2019).

En el caso que nos ocupa sería necesario el desarrollo de esquemas de análisis de riesgo consensuados por expertos que integren las características particulares de los ambientes agrícolas, ya que son, en un gran número de ocasiones, puerta de entrada al establecimiento de poblaciones de especies exóticas potencialmente invasoras (Richardson et al., 2000). Por otro lado, entre los aspectos a considerar en dichos esquemas resulta necesario, por un lado, una valoración más exhaustiva de los atributos funcionales de las especies ya que éstos son claves en el éxito de su establecimiento en campos de cultivo. Asimismo sería necesario valorar aquellas prácticas de manejo que favorecen la invasibilidad de los agroecosistemas. Una integración de dichos esquemas dentro de un análisis global de la vulnerabilidad de cada sistema agrícola permitiría desarrollar una metodología mucho más robusta de evaluación del potencial in- vasor de nuevas especies exóticas, así como de manejo de los cultivos de cara a la priorización, monitorización y erradicación de estas especies una vez presentes en ellos.

En este sentido, dichas estrategias de prevención deben ser aplicadas, no sólo en el análisis del riesgo que suponen las especies vegetales exóticas detectadas en nuestros cultivos, sino en la evaluación de la probabilidad de entrada y establecimiento de aquellas otras que, aun sin haber sido citadas todavía en nuestro entorno, ya han demostrado un comportamiento invasor en territorios cercanos o en hábitats de características similares. Se trata de establecer medidas de prevención al igual que se llevan a cabo contra patógenos y plagas agrícolas, no solo por ser la estrategia más eficaz, sino también la opción más económica (Andreu y Vilà, 2007). Esta anticipación requiere no solo la categorización de las especies potencialmente invasoras, sino también el conocimiento de las características ambientales y de adaptación al manejo que favorecen su expansión en las regiones de origen o en aquellas otras donde ya se han establecido (Pheloung et al., 1999).

Para alcanzar la máxima efectividad de estas estrategias, en el contexto de los sistemas agrícolas, resulta necesario e imprescindible la colaboración de distintas entidades y organismos gestores, tanto a nivel local como regional, así como de una adecuada formación, en el ámbito de las invasiones biológicas, de los actores y agentes involucrados (técnicos de Asociaciones de Defensa Vegetal, asesores cualificados, técnico de Servicios de Sanidad Vegetal, etc.). Todos estos elementos, acompañados de campañas de sensibilización son prioritarios de cara a reforzar el apoyo que los agricultores afectados deban recibir de las políticas de prevención y gestión de las malas hierbas invasoras. 


\section{Referencias bibliográficas}

Andreu J, Vilà M (2007). Análisis de la gestión de las plantas exóticas en los espacios naturales españoles. Ecosistemas 16: 109-124.

BOE (2002). Ley 43/2002, de 20 de noviembre, de sanidad vegetal (última modificación 5 de marzo de 2011). Boletín Oficial del Estado núm. 279, de 21 de noviembre de 2002.

BOE (2007). Ley 42/2007, de 13 de diciembre, del Patrimonio Natural y de la Biodiversidad (última actualización 21 de julio de 2018). Boletín Oficial del Estado núm. 299, de 14 de diciembre de 2007.

BOE (2011). Real Decreto 1628/2011, de 14 de noviembre, por el que se regula el listado y catálogo español de especies exóticas invasoras. Boletín Oficial del Estado núm. 298, de 12 de diciembre de 2011. Disposición derogada.

BOE (2013). Real Decreto 630/2013, de 2 de agosto, por el que se regula el Catálogo español de especies exóticas invasoras. Boletín Oficial del Estado núm. 185, de 5 de agosto de 2013.

BOE (2016). Sentencia de 16 de marzo de 2016, de la Sala Tercera del Tribunal Supremo, que anula los siguientes extremos del Real Decreto 630/2013, de 2 de agosto, que regula el Catálogo español de especies exóticas invasoras [...]. Boletín Oficial del Estado núm. 146, de 17 de junio de 2016.

Carretero JL (2004) Flora arvense española. Las malas hierbas de los cultivos españoles. Ed. Phytoma España.

Clements DR, DiTommaso A, Jordan N, Booth BD, Cardina J, Doohan D, Mohler CL, Murphy SD, Swanton CL (2004). Adaptability of plants invading North American cropland. Agriculture Ecosystems and Environment 104: 379-398. https://doi.org/10.1016/j.agee.2004.03.003

Conesa JA, Sanz-Elorza M (1998). Azolla filliculoides Lam y Heteranthera limosa (Sw.) Willd, dos nuevas malas hierbas de los arrozales altoaragoneses. ITEA-Información Técnica Económica Agraria 94V(3): 177-184.

Crosti R, Cascone C, Cipollaro S (2010). Use of a weed risk assessment for the Mediterranean re- gion of Central Italy to prevent loss of functionality and biodiversity in agro-ecosystems. Biological Invasions 12: 1607-1616. https://doi.org/ 10.1007/s10530-009-9573-6

Daehler CC (1998). The taxonomic distribution of invasive angiosperms plants: ecological insights and comparison to agricultural weeds. Biological Conservation 84: 167-180. https://doi.org/ 10.1016/S0006-3207(97)00096-7

Dana ED, García-de-Lomas J, Verloove F, Vilà M (2019). Common deficiencies of actions for managing invasive alien species: a decision-support checklist. NeoBiota 48: 97-112. https://doi.org/ 10.3897/neobiota.48.35118

Davis MA, Grime JP, Thompson K (2000). Fluctuating resources in plant communities: a general theory of invasibility. Journal of Ecology 88: 528-534. https://doi.org/10.1046/j.1365-2745. 2000.00473.x

D'hondt B, Vanderhoeven S, Roelandt S, Mayer F, Versteirt V, Adriaens T, Ducheyne E, Martin GS, Grégoire JC, Stiers I, Quoilin S, Cigar J, Heughebaert A, Branquart E (2015). Harmonia+ and Pandora': risk screening tools for potentially invasive plants, animals and their pathogens. Biological Invasions 17: 1869-1883. https://doi.org/ 10.1007/s10530-015-0843-1

Díaz A, Taberner A, Vilaplana L (2019). The emergence of a new weed in maize plantations: characterization and genetic structure using microsatellite markers. Genetic Resources and Crop Evolution (publicado on line el 13 septiembre 2019). https://doi.org/10.1007/s10722-01900828-z

DOCE (2000). Directiva 2000/29/CE del Consejo, de 8 de mayo de 2000, relativa a las medidas de protección contra la introducción en la Comunidad de organismos nocivos para los vegetales o productos vegetales y contra su propagación en el interior de la Comunidad.

DOGC (1985). Decret 6/1985, de 14 de gener, sobre mesures urgents per a l'eradicació de focus de plagues especialment perilloses per als vegetals. Diari Oficial de la Generalitat de Catalunya núm. 511, de 8 de febrero de 1985.

DOGC (2005). Ordre ARP/10/2005, de 18 de gener, per la qual es declara l'existència oficial de la 
mala herba Sicyos angulatus L, i s'estableixen mesures obligatòries de Iluita. Diari Oficial de la Generalitat de Catalunya núm. 4315, de 3 de febrero de 2005.

DOGC (2006a). Ordre ARP/339/2006, de 30 de juny, per la qual es declara l'existència oficial de la mala herba Solanum carolinense, i s'estableixen mesures obligatòries de Iluita. Diari Oficial de la Generalitat de Catalunya núm. 4671, de 7 de julio de 2006.

DOGC (2006b). Ordre ARP/342/2006, de 30 de juny, per la qual es declara l'existència oficial a Catalunya de les males herbes Leptochloa sp., Leersia oryzoides i Sagittaria sp., i s'estableixen mesures obligatòries de Iluita. Diari Oficial de la Generalitat de Catalunya núm. 4671, de 7 de julio de 2006.

DOGC (2014). Decret 137/2014, de 7 d'octubre, sobre mesures per evitar la introducció i propagació d'organismes nocius especialment perillosos per als vegetals i productes vegetals. Diari Oficial de la Generalitat de Catalunya núm. 6724, de 9 de octubre de 2014.

DOGC (2019). Ordre ARP/172/2019, de 10 de setembre, per la qual es declara l'existència de la mala herba Amaranthus palmeri i es qualifica d'utilitat pública la lluita contra aquesta. Diari Oficial de la Generalitat de Catalunya núm. 7959, de 13 de septiembre de 2019.

DOUE (2014). Reglamento (UE) 1143/2014 del Parlamento Europeo y del Consejo, de 22 de octubre de 2014, sobre la prevención y la gestión de la introducción y propagación de especies exóticas invasoras.

DOUE (2016). Reglamento de Ejecución (UE) 2016/ 1141 de la Comisión, de 13 de julio de 2016, por el que se adopta una lista de especies exóticas invasoras preocupantes para la Unión de conformidad con el Reglamento (UE) $n^{\circ} 1143 / 2014$ del Parlamento Europeo y del Consejo.

DOUE (2017). Reglamento de Ejecución (UE) 2017/ 1263 de la Comisión, de 12 de julio de 2017, por el que se actualiza la lista de especies exóticas invasoras preocupantes para la Unión establecida por el Reglamento de Ejecución (UE) 2016/1141 de conformidad con el Reglamento (UE) $n^{\circ} 1143 / 2014$ del Parlamento Europeo y del Consejo.
EPPO (2019). European and Mediterranean Plant Protection Organization. EPO Data Base. Disponible en: www.eppo.int/ACTIVITIES/invasive_alien_plants/iap_lists (Consultado: 19 de julio de 2019).

Essl F, Nehring S, Klingenstein F, Milasowszky N, Nowack C, Rabitsch W (2011). Review of risk assessment systems of IAS in Europe and introducing the German-Austrian Black List Information System (GABLIS). Journal for Nature Conservation 19: 339-350. https://doi.org/10. 1016/j.jnc.2011.08.005

EXOCAT (2019). Exocat, Sistema d'Informació de les Espècies Exòtiques de Catalunya. CREAF y Generalitat de Catalunya. Disponible en: http:// exocat.creaf.cat (Consultado: 23 de julio de 2019).

Gassó N, Sol D, Pino J, Dana ED, Lloret F, SanzElorza M, Sobrino E, Vilà M (2009). Exploring species attributes and site characteristics to assess plant invasions in Spain. Diversity and Distributions 15: 50-58. https://doi.org/10.1111/ j.1472-4642.2008.00501.x

González-Moreno P, Lazzaro L, Vilà M, Preda C, Adriaens T, Bacher S, Brundu G, Copp GH, Essl F, García-Berthou E, Katsanevakis S, Moen TL, Lucy FE, Nentwig W, et al. (2019). Consistency of impact assessment protocols for non-native species. NeoBiota 44: 1-25. https://doi.org/ 10.3897/neobiota.44.31650

Hejda M, de Bello F (2013). Impact of plant invasions on functional diversity in the vegetation of Central Europe. Journal of Vegetation Science 24: 890-897. https://doi.org/10.1111/jvs.12026

Hulme PE (2012). Weed risk assessment: A way forward or a waste of time? Journal of Applied Ecology 49: 10-19. https://doi.org/10.1111/j. 1365-2664.2011.02069.x

IUCN (2000). Guidelines for the Prevention of Biodiversity Loss Caused by Alien Invasive Species. IUCN, Gland, Switzerland.

Juárez-Escario A, Valls J, Solé-Senan XO, Conesa JA (2013). A plant-traits approach to assessing the success of alien weed species in irrigated Mediterranean orchards. Annals of Applied Biology 162: 200-213. https://doi.org/10.1111/ aab.12012 
Juárez-Escario A, Conesa JA, Solé-Senan X (2016). Identifying alien plants linkages between irrigated orchards and adjacent riparian habitats from a trait-based approach. Agriculture, Ecosystems and Environment 225: 173-183. https:// doi.org/10.1016/j.agee.2016.04.015

Juárez-Escario A, Solé-Senan XO, Recasens J, Taberner A, Conesa JA (2018). Long-term compositional and functional changes in alien and native weed communities in annual and perennial irrigated crops. Annals of Applied Biology 173: 42-54. https://doi.org/10.1111/aab.12432

Keller RP, Lodge DM, Finnoff DC (2007). Risk assessment for invasive species produces net bioeconomic benefits. Proceedings of the National Academy of Sciences 104: 203-207. https://doi. org/10.1073/pnas.0605787104

Kenis M, Bacher S, Baker RHA, Branquart E, Brunel S, Holt J, Hulme PE, MacLeod A, Pergl J, Petter F, Pyšek P, Schrader G, Sissons A, Starfinger U, Schaffner U (2012). New protocols to assess the environmental impact of pests in the EPPO decision-support scheme for pest risk analysis. EPPO Bulletin 42: 21-27. https://doi.org/10.1111/ j.1365-2338.2012.02527.x

$K$ ivánek $M$, Pyšek $P$ (2006). Predicting invasions by woody species in a temperate zone: a test of three risk assessment schemes in the Czech Republic (Central Europe). Diversity and Distributions 12: 319-327. https://doi.org/10.1111/j. 1366-9516.2006.00249.x

Langa R, Aibar J, Cirujeda A, Mari Al, León M, y Pardo G (2015). Estudio de la resistencia de Conyza spp. al herbicida glifosato en Aragón. XV Congreso de la Sociedad Española de Malherbología: La Malherbología y la transferencia tecnológica, 19-22 octubre 2015, Sevilla. pp. 99-105.

Nentwig W, Bacher S, Pyšek P, Vilà M, Kumschick $S$ (2016) The generic impact scoring system (GISS): a standardized tool to quantify the impacts of alien species. Environmental Monitoring and Assessment 188: 315. https://doi.org/ 10.1007/s10661-016-5321-4

Osca JM (2013). Expansion of Leptochloa fusca ssp. uninervia and Leptochloa fusca ssp. fascicularis in rice fields in Valencia, eastern Spain. Weed Research 53: 479-488. https://doi.org/ 10.1111/wre.12046
Pál R (2004). Invasive plants threaten segetal weed vegetation of South Hungary. Weed Technology 18: 1314-1318. https://doi.org/10.1614/0890037X(2004)018[1314:IPTSWV]2.0.CO;2

Pheloung PC, Williams PA, Halloy SR (1999). A weed risk assessment model for use as a biosecurity tool evaluating plant introductions. Journal of Environmental Management 57: 239-251. https://doi.org/10.1006/jema.1999.0297

Pardo G, Fuertes S, Fernández-Cavada S, Betrán E, Cirujeda A, Marí Al, Aibar J, Zaragoza C, Perdiguer A, Llenes JM, Montull JM, Taberner A (2015). Presencia de teosinte (Zea spp.) como mala hierba en los regadíos del valle del Ebro. XV Congreso de la Sociedad Española de Malherbología: La Malherbología y la transferencia tecnológica, 19-22 octubre 2015, Sevilla, pp. 417-424.

Pimentel D, Zuniga R, Morrison D (2005). Update on the environmental and economic costs associated with alien-invasive species in the United States. Ecological Economics 52: 273-288. https://doi.org/10.1016/j.ecolecon.2004.10.002

Protopopova VV, Shevera MV, Mosyakin SL (2006) Deliberate and unintentional introduction of invasive weeds: A case study of the alien flora of Ukraine. Euphytica, 148: 17-33. https://doi.org/ 10.1007/s10681-006-5938-4

Pyšek P, Richardson DM, Rejmánek M, Webster GL, Williamson M, Kirschner J (2004). Alien plants in checklists and floras: towards better communication between taxonomists and ecologists. Taxon 53: 131-143. https://doi.org/ $10.2307 / 4135498$

Pyšek P, Lamdon PW, Arianoutsou M, Kühn I, Pino J, Winter M (2009). Alien vascular plants of Europe. En: Handbook of Alien Species in Europe, pp. 43-61. Ed. Springer Science. https://doi.org/ 10.1007/978-1-4020-8280-1_4

Pyšek P, Chytrý M, Jarošík V (2010). Habitats and land-use as determinants of plant invasions in the temperate zone of Europe. En: Bioinvasions and globalization: ecology, economics, management and policy (Ed. Perrings $C$, Mooney $\mathrm{H}$ and Williamson $\mathrm{M}$ ), pp. 66-79. Oxford University Press, Oxford. https://doi.org/10.1093/ acprof:oso/9780199560158.003.0006 
Recasens J, Conesa JA (1995). Nuevas malas hierbas alóctonas en los cultivos de regadío de Cataluña. Actas Congreso 1995 Sociedad Española de Malherbología, 14-16 noviembre 1995, Huesca, pp. 59-65.

Recasens J, Conesa JA (2003). Atributs biològics de la flora arvense al-lòctona de Catalunya. Acta Botanica Barcinonensia 48:45-46.

Recasens J, Calvet V, Cirujeda A, Conesa JA (2005). Phenological and demographic behaviour of an exotic invasive weed in agroecosystems. Biological Invasions 7: 17-27. https://doi.org/10. 1007/s10530-004-9625-x

Recasens J, Conesa JA, Millán J, Taberner A (2007). Previsión del impacto agronómico y económico de Sicyos angulatus como mala hierba invasora de campos de maíz en Cataluña. Actas del Congreso 2007 de la Sociedad Española de Malherbología, 7-9 noviembre 2007, Albacete, España, pp. 343-348.

Recasens J, Conesa JA (2011). Presencia de la mala hierba Amaranthus palmeri en el NE de la península Ibérica. Una amenaza como potencial invasora de cultivos extensivos de regadío. Boletín de Sanidad Vegetal. Plagas 37: 129-132.

Recasens J, Osuna MD, Royo-Esnal A, Torra J (2017). Amaranthus palmeri en Cataluña y Aragón. ¿Tres poblaciones con un mismo origen? Actas XVI Congreso de la Sociedad Española de Malherbología, 24-27 octubre 2017, Pamplona, pp. 15-20.

Richardson DM, Pyšek $P$, Rejmánek $M$, Barbour MG, Panetta DF, West CJ (2000). Naturalization and invasion of alien plants: concepts and definitions. Diversity and Distributions 6: 93-107. https://doi.org/10.1046/j.1472-4642.2000. 00083.x

Richardson DM, Pyšek P (2006). Plant Invasions: merging the concepts of species invasiveness and community invasibility. Progress in Physical Geography 30: 409-431. https://doi.org/10.1191/ 0309133306pp490pr
Sanz-Elorza M, Dana E, Sobrino E (2004). Atlas de las Plantas Alóctonas Invasoras en España. Dirección General para la Biodiversidad. Ministerio de Medio Ambiente. Madrid. 384 pp.

Sanz-Elorza MG, Mateo RG, González Bernardo F (2009). The historical role of agriculture and gardening in the introduction of alien plants in the western Mediterranean. Plant Ecology 202: 247-256. https://doi.org/10.1007/s11258-0089474-2

Shea K, Chesson P (2002). Community ecology theory as a framework for biological invasions. Trends in Ecology and Evolution 17: 170-176. https://doi.org/10.1016/S0169-5347(02)02495-3

Sellers BA, Smeda RJ, Johnson WG, Kending JA, Ellersieck MR (2003). Comparative growth of six Amaranthus species in Missouri. Weed Technology 51: 329-313. https://doi.org/10.1614/00431745(2003)051[0329:CGOSAS]2.0.CO;2

Taberner A, Sans M (2005). Procedimiento de erradicación de Sicyos angulatus L. en maíz. En: Malherbología Ibérica y Magrebí: Soluciones comunes a problemas comunes (Ed. Menéndez J, Bastida F, Fernández-Quintanilla C, GonzálezAndújar JL, Recasens J, Royuela M, Verdú, Zaragoza C) pp. 569-574. Universidad de Huelva.

Vilà J (1997). Presencia de Leersia oryzoides (L.) Sw. en los arrozales del Baix Empordà. Boletín de la Sociedad Española de Malherbología 24: 9

Vilà M, Williamson M, Londsale M (2004). Competition experiments on alien weeds with crops: lessons for measuring plants invasions impact? Biological invasions 6: 59-69. https:// doi.org/10.1023/B:BINV.0000010122.77024.8a

Zaragoza C, García Floria MC, Aibar J (1993). Presencia de Heteranthera reniformis Ruíz y Pavón en el cultivo de arroz en Huesca. Actas Congreso 1993 Sociedad Española de Malherbología, 1-3 septiembre 1993, Lugo, pp. 37-40.

(Aceptado para publicación el 21 de octubre de 2019) 